\title{
CONCURRENT FEED-FORWARD/FEED-BACK DESIGN FOR FLEXIBLE STRUCTURES
}

\author{
Marco Muenchhof \\ Graduate Student \\ Institute of Automatic Control \\ University of Technology at Darmstadt, Darmstadt, 64283 \\ GERMANY \\ Tarunraj Singh \\ Associate Professor \\ Dept. of Mech. \& Aero. Eng., \\ University of Buffalo, Buffalo, NY 14260
}

\begin{abstract}
This paper discusses the concurrent design of a feedforward time-delay filter and a linear state feed-back controller. The optimization is carried out using a quadratic cost functional which weights both, disturbance rejection and control tracking performance. A minimax optimization scheme is employed to achieve robustness with respect to parameter deviations. Analytical gradients are provided for the cost function. This information can be exploited to expedite convergence of the optimization procedure. The proposed technique is illustrated on the Floating Oscillator benchmark problem.
\end{abstract}

\section{INTRODUCTION}

Vibration control has been subject to intense research, aiming at applications as diverse as maneuvering of large space structures, ${ }^{1}$ flexible arm robots, ${ }^{2}$ computer disk drives, ${ }^{3}$ and cranes. ${ }^{4}$ In most of these applications, the goal is to minimize the maneuver time, while ensuring quiescent final states. During this exhaustive research, multifarious constraints have been added to the problem formulation, such as limits on the fuel consumed, ${ }^{5}$ robustness to modelling uncertainties, ${ }^{67}$ and maximum permitted deformation. ${ }^{8}$

Traditionally, there are two approaches to the control of flexible structures, either avoiding excitations by shaping the control input appropriately or damping vibration by the application of a suitable feed-back control law. As separate entities, both of these approaches have been exhaustively investigated and a variety of solutions have been developed. These approaches will be briefly reviewed.

One specific method of forming the control input which does not excite vibrations is by the use of timedelay filters. This technique was initially developed by Smith $^{9}$ in 1957 and was termed "Posicast". It is based on dividing a step input into a number of spaced excitations, which are chosen such that there is no remanent vibrational energy at the end of the maneuver. In this initial work, robustness issues had not been considered. In 1990, Singer and Seering ${ }^{6}$ suggested a $^{2}$ practice to design input shapers addressing the sensitivity issues. All these techniques are based in the time domain. Singh and Vadali ${ }^{7}$ pointed out that a time delay filter which cancels the underdamped poles of a system results in the same control profile as the input shaping controller. Thus, input prefilters can also be designed using frequency domain techniques and properties.

Recently, other techniques have been developed to address the issue of desensitizing the controller to modelling errors. Singh ${ }^{10}$ proposed a minimax formulation to desensitize the input-preshaping controller with respect to modelling errors. Muenchhof and Singh ${ }^{11}$ considered limitations on the time rate of change of the control input for both single-input and multi-input systems. They illustrate the reduction of energy in the power spectrum at the higher frequencies, which is desirable for flexible structures. The tutorial by Singh and Singhose ${ }^{12}$ summarizes the results obained in this area of research.

A variety of design methods for feed-back control systems have been developed for both linear and nonlinear systems. For linear systems, the linear state feed-back is the prevailing control setup due to its ease of application and the multitude of applicable design methods such as pole placement or the linear quadratic regulator setting. ${ }^{13}$ The latter technique, abbreviated LQR, allows for the design of linear state feed-back controllers which minimize a quadratic cost function weighting both deviation of the states from the desired final value and the control effort expended. Many methods have been proposed which extend the basic idea behind the linear quadratic regulator. Among them are methods to robustify the regulator with respect to parametric uncertainties of the plant and 
methods to design regulators for plants subject to white or colored noise excitation. Mills and Bryson ${ }^{14}$ proposed a minimax technique, where the cost function is evaluated not only for the nominal set of system parameters but also for a number of perturbed systems. They also presented this design technique for white noise disturbances, designing a linear-quadraticgaussian controller with parameter robustness. ${ }^{15}$

The advantages of the two aforementioned approaches complement one another. On the one hand, it is much better to prevent residual vibration by using a feed-forward control scheme than trying to eliminate these vibrations once they came into existence. On the other hand, feed-forward controllers cannot cope with unmeasured disturbances. This is where the feed-back controller can show its full potential. Those considerations lead to the idea of using a hybrid control approach. Different controller structures evolve depending on whether the input-prefilter is also part of the feed-back loop ${ }^{16}$ or not.

In this paper, a controller structure consisting of a time-delay filter and linear state feed-back is considered. The input prefilter is not part of the feed-back loop. Quadratic cost functions are used to rate the control tracking performance as well as the disturbance rejection capabilities. The controller design has been posed as a minimax optimization problem. This permits increasing the robustness of the controller with respect to parametric uncertainties. Statistical information about the uncertain parameters, such as lower bounds, upper bounds, and the expected value can easily be included. The level of insensitivity can be increased arbitarily, trading maneuver time for robustness.

This paper is divided as follows: First, the problem is formulated and the cost functions are described. This is followed by the development of analytical gradients for the cost functions. Next, the minimax approach is illustrated. Finally, numerical examples are presented, which detail the performance of the new control technique for the Floating Oscillator standard benchmark problem. ${ }^{17}$

\section{PROBLEM FORMULATION}

The paper discusses the design of a combined feedforward/feed-back controller for linear systems. Linear systems can be described by the first order differential equation

$$
\begin{aligned}
\dot{x}(t) & =P(p) x(t)+Q(p) u(t)+Q_{w} w(t) \\
y(t) & =R(p) x(t)
\end{aligned}
$$

where $P$ is the state matrix, $Q$ the input distribution matrix and $R$ the output matrix. It is assumed that there is no direct feed-through from the input to the output. The parameters of the system are uncertain, which means that they depend on the uncertain vector $p$, bounded by

$$
p_{L} \leq p \leq p_{U}
$$

In addition to these parametric uncertainties, the system is also subject to a white noise disturbance $w(t)$, which is acting on the states as described by $Q_{w}$.

The system is controlled by a time-delay filter which is described by the transfer function

$$
G_{F}(s)=\sum_{k=1}^{N} A_{k} e^{-s T_{k}},
$$

whwre $T_{1}=0$. This feed-forward controller is augmented by a linear state-feed-back-controller, which has the form

$$
u(t)=-K x(t) .
$$

The system setup is depicted in Fig. 1.

The engineer designing a control system has two goals: Rejection of disturbances and fast control action. To accomodate these demands, a weighted combination of two cost functions will be chosen. The first cost function rates the control performance. Here, the residual energy was chosen, thus

$$
\begin{aligned}
F_{R E} & =\frac{1}{2} x^{T}(t) X x(t) \\
& +\frac{1}{2}\left(x(t)-x_{\text {final }}\right)^{T}(t) Y\left(x(t)-x_{\text {final }}\right),
\end{aligned}
$$

where $x_{\text {final }}$ is the desired final value of the states. The equation is evaluated for $t=T_{\text {Eval }}$. The last term had to be inserted to account for rigid body modes, i. e. modes, which do not necessarily have to return to zero at the end of the maneuver. It is suggested to choose $X$ as

$$
X=\left[\begin{array}{cc}
K_{\text {sys }} & 0 \\
0 & M_{\text {sys }}
\end{array}\right],
$$

where $K_{\text {sys }}$ is the system's stiffness matrix and $M_{\text {sys }}$ the corresponding mass matrix. Furthermore, $Y$ is set up as

$$
Y=\left[\begin{array}{cc}
K_{\text {hyp }} & 0 \\
0 & 0
\end{array}\right]
$$

where $K_{\text {hyp }}$ is a diagonal matrix, which contains the "spring stiffness" of hypothetical springs, which are thought to be connected to rigid body modes of the structure and would be deformed as long as the rigid body mode has not reached its desired final displacement. This way, the rigid body mode does also turn up in the cost function. Upon this selection of $X$ and $Y$, the quadratic cost can easily be interpreted as the sum of potential and kinetic energy stored in the system at the time instant $T_{\text {Eval }}$. This snapshot of the energy distribution is a measure of the amount of residual vibration. The time of evaluation, $T_{\text {Eval }}$, can be chosen in two ways. First, it can be chosen such that $T_{\text {Eval }}=$ const, in which case the cost function is evaluated at a fixed instant in time located somewhere 


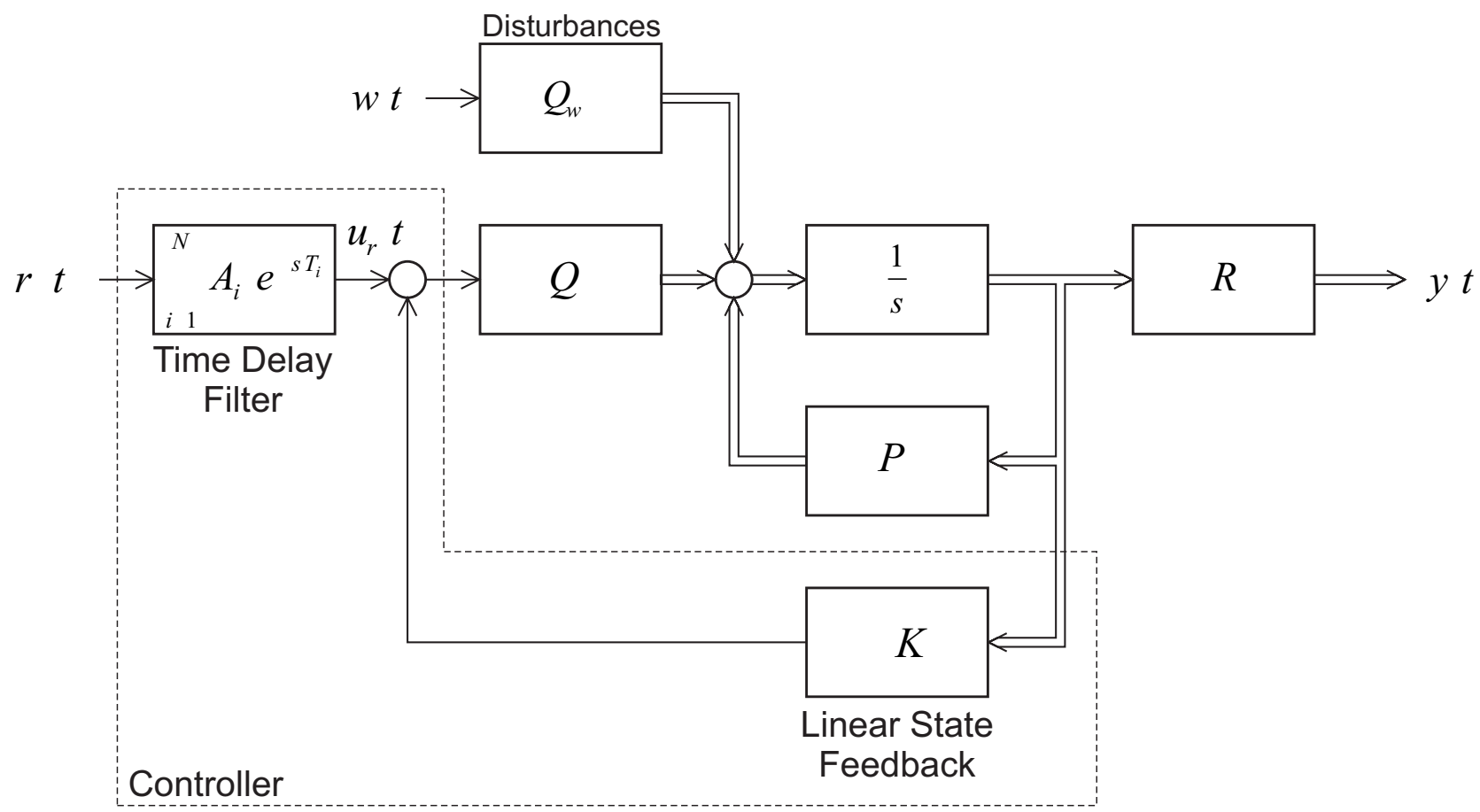

Fig. 1 System Setup

after the end of the maneuver. The second case is to evaluate the cost function always at the end of the maneuver, for which case $T_{E v a l}=T_{n}$. In this paper, the former approach is chosen.

The second cost function was chosen as the expected value of the quadratic cost for the system being subjected to white noise $w(t)$. The cost function is given as the expected value of the time integral

$$
F_{N}=\mathcal{E}\left[\lim _{t_{f} \rightarrow \infty} \frac{1}{2 t_{f}} \int_{0}^{t_{f}}\left(y^{T} B y+u^{T} D u\right) d t\right]
$$

with the cost matrix $B$ penalizing the deviation of states, the cost matrix $D$ weighting the control effort and $\mathcal{E}[\ldots]$ is the expectation operator.

The closed loop dynamics are determined by the state matrix $\tilde{P}$, given as

$$
\tilde{P}=P-Q K
$$

The cost function in Eq. 8 is calculated using the LQR theory. Here, the cost can be calculated by first solving the Lyapunov equation ${ }^{14}$

$$
\tilde{P} Z+Z \tilde{P}^{T}+Q_{W} W Q_{W}^{T}=0 .
$$

Here, $W$ is the covariance matrix of the noise and $Z$ is the solution of the Lyapunov equation, which will then be used to calculate the cost functional

$$
F_{N}=\operatorname{tr}(Z S)
$$

where

$$
S=R^{T} B R+K^{T} D K
$$

The total cost is given as a linear combination of the individual cost functions,

$$
F=\alpha F_{R E}+(1-\alpha) F_{N}
$$

where the factor $\alpha$ with $0<\alpha<1$ weights the two cost functions. The two extremal cases represent the design of either only a robust linear state-feed-back controller or only a robust time-delay filter. For the case $\alpha=0$, the design variables which define the time delay filter do not turn up in the cost function. For $\alpha=1$, the effect of noise is not considered.

\section{ANALYTICAL GRADIENTS}

In this section, analytical gradients are provided for both cost functions. For the residual energy, given in Eq. 5, the first derivative with respect to the amplitudes of the time-delay filter is given as

$$
\begin{aligned}
\frac{\partial F_{R E}(t)}{\partial A_{i}} & =x^{T}(t) X \frac{\partial x(t)}{\partial A_{i}} \\
+ & \left(x(t)-x_{\text {final }}\right)^{T}(t) Y \frac{\partial x(t)}{\partial A_{i}}
\end{aligned}
$$

and requires knowledge of the derivative of the states with respect to the time delay filter amplitudes. These derivatives can easily be calculated as

$$
\frac{\partial x(t)}{\partial A_{i}}=\int_{T_{i}}^{t} e^{\tilde{P}(t-\tau)} Q d \tau \text { if } t \geq T_{i}
$$


Next, the derivative with respect to the switching times is determined,

$$
\begin{aligned}
\frac{\partial F_{R E}(t)}{\partial T_{i}} & =x^{T}(t) X \frac{\partial x(t)}{\partial T_{i}} \\
& +\left(x(t)-x_{\text {final }}\right)^{T}(t) Y \frac{\partial x(t)}{\partial T_{i}}
\end{aligned}
$$

which necessitates calculation of the first derivative of the states evaluated at $T_{\text {Eval }}$ with respect to the switching times, which is found to be

$$
\left.\frac{\partial x}{\partial T_{i}}\right|_{t=T_{E v a l}}=-A_{i} e^{\tilde{P}\left(T_{E v a l}-T_{i}\right)} Q .
$$

In order to calculate the derivative

$$
\begin{aligned}
\frac{\partial F_{R E}(t)}{\partial k_{i}} & =x^{T}(t) X \frac{\partial x(t)}{\partial k_{i}} \\
& +\left(x(t)-x_{\text {final }}\right)^{T}(t) Y \frac{\partial x(t)}{\partial k_{i}}
\end{aligned}
$$

the partial derivative of the states with respect to the controller gains must be determined. An augmented state space system is constructed as

$$
\left[\begin{array}{c}
\dot{x}(t) \\
\frac{\partial \dot{x}(t)}{\partial k_{i}}
\end{array}\right]=\left[\begin{array}{cc}
P-Q K & 0 \\
-Q \frac{\partial K}{\partial k_{i}} & P-Q K
\end{array}\right]\left[\begin{array}{c}
x(t) \\
\frac{\partial x(t)}{\partial k_{i}}
\end{array}\right]+\left[\begin{array}{c}
Q \\
0
\end{array}\right] u_{r}(t) .
$$

The newly introduced state variables contain the derivative of the system states with respect to the controller gain $k_{i}$. Solving this differential equation up to time $T_{\text {Eval }}$ yields the derivative of the states with respect to the controller gains at the time instant $T_{E v a l}$.

For the second cost function, which is a measure of the system dynamics due to excitation at input $w(t)$, the gradients are easier to calculate. This cost function depends neither on $A_{i}$ nor on $T_{i}$. The only derivatives to be calculated are the ones with respect to the controller gains. They can be obtained by

$$
\frac{\partial F_{N}}{\partial k_{i}}=\operatorname{tr}\left(\frac{\partial Z}{\partial k_{i}} S+Z \frac{\partial S}{\partial k_{i}}\right),
$$

where $Z_{i}=\frac{\partial Z}{\partial k_{i}}$ is the solution of the Lyapunov equation

$$
\tilde{P}^{T} Z_{i}+Z_{i} \tilde{P}+\frac{\partial \tilde{P}}{\partial k_{i}} Z+Z\left(\frac{\partial \tilde{P}}{\partial k_{i}}\right)^{T}=0
$$

The partial derivative of $S$ with respect to $k_{i}$ is given as

$$
\frac{\partial S}{\partial k_{i}}=2\left(\frac{\partial K}{\partial k_{i}}\right)^{T} D K
$$

Since the final cost function is a weighted linear combination of the individual cost functions, the derivatives of the entire cost function are simply a linear combination of the individual derivatives.

\section{INCREASING ROBUSTNESS}

The goal of this paper is to design a concurrent feedforward/feed-back controller with increased robustness towards parameter deviations. This is achieved by a minimax approach,

$$
\min _{\{K, T, A\}} \max _{p} F(K, T, A, p, \alpha)
$$

The cost function is evaluated not only at the nominal point, but also at the corners of the uncertain space, which is bounded by $p_{L}$ and $p_{U}$. The optimization algorithm starts off with a controller designed for the nominal system. This initial feasible solution can easily be calculated since many methods already exist for both linear state feed-back controllers and timedelay filters provided only the nominal parameter set is taken into account and the problems are looked at separately. Once this initial guess has been obtained, the algorithm starts to look at the performance of all perturbed systems in addition to the performance of the nominal system. The bounding box of the uncertain space, which was originally limited to just one point, namely the nominal parameter set, is now repeatedly increased until the total uncertain space is covered. This method is depicted in Fig. 2 for a system with three uncertain parameters.

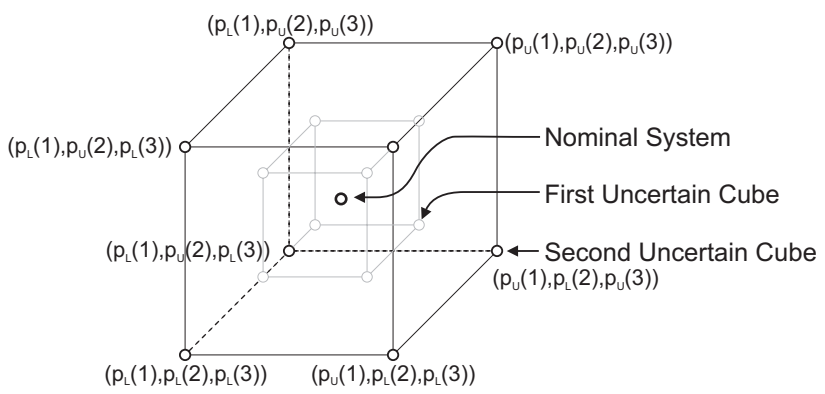

Fig. 2 Relaxing Bounds of the Uncertain Space Considered

\section{NUMERICAL EXAMPLES}

The plant under consideration in this section on numerical examples is the Floating Oscillator, a two-mass/spring-and-damper system as illustrated in Fig. 3. This plant represents a standard benchmark problem with one rigid body mode and one flexible mode. ${ }^{17}$ The system's dynamics are governed by

$$
\begin{aligned}
& {\left[\begin{array}{cc}
m_{1} & 0 \\
0 & m_{2}
\end{array}\right]\left[\begin{array}{l}
\ddot{y}_{1}(t) \\
\ddot{y}_{2}(t)
\end{array}\right]+\left[\begin{array}{cc}
c & -c \\
-c & c
\end{array}\right]\left[\begin{array}{l}
\dot{y}_{1}(t) \\
\dot{y}_{2}(t)
\end{array}\right]} \\
& +\left[\begin{array}{cc}
k & -k \\
-k & k
\end{array}\right]\left[\begin{array}{l}
y_{1}(t) \\
y_{2}(t)
\end{array}\right]=\left[\begin{array}{l}
1 \\
0
\end{array}\right] u(t)+\left[\begin{array}{l}
1 \\
0
\end{array}\right] u_{w}(t) .
\end{aligned}
$$

The plant has four uncertain parameters, which are the two masses $m_{1}$ and $m_{2}$, the spring stiffness $k$ and the damping coefficient $c$, represented by the uncertain vector $p$. The nominal system parameters are listed in Table 1 along with their respective uncertainty. 
Table 1 Nominal System Parameters

\begin{tabular}{r|c|r|r} 
Number & Parameter & Value & Uncertainty \\
\hline 1 & $k$ & 1.0 & $\pm 1.0 \sigma$ \\
\hline 2 & $c$ & 0.1 & $\pm 0.1 \sigma$ \\
\hline 3 & $m_{1}$ & 1.0 & $\pm 1.0 \sigma$ \\
\hline 4 & $m_{2}$ & 1.0 & $\pm 1.0 \sigma$
\end{tabular}

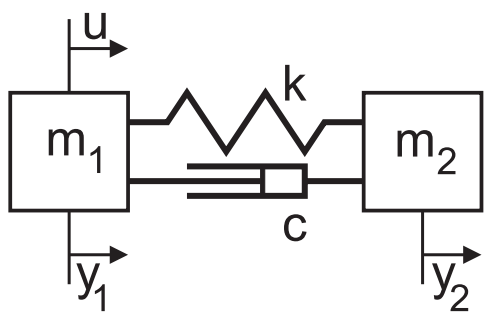

Fig. 3 Floating Oscillator With Damping

Table 2 Control Profile Parameters for $\alpha=0.9$ and $\sigma=20 \%$

\begin{tabular}{c|c|c|r|r} 
& Nom & Rob & 1 TDF & 2 TDF \\
\hline$k_{1}$ & 0.7669 & 0.8184 & 0.8203 & 0.8205 \\
\hline$k_{2}$ & 0.2331 & 0.1832 & 0.1844 & 0.1847 \\
\hline$k_{3}$ & 1.2298 & 1.1350 & 1.1346 & 1.1354 \\
\hline$k_{4}$ & 1.1226 & 1.0168 & 1.0159 & 1.0167 \\
\hline$T_{1}$ & - & - & 1.9580 & 2.0668 \\
\hline$A_{1}$ & - & - & 0.4133 & 0.4674 \\
\hline$T_{2}$ & - & - & - & 4.3328 \\
\hline$A_{2}$ & - & - & - & 0.1819 \\
\hline
\end{tabular}

For $\alpha=0.9$, different controllers will now be designed. For reasons of comparison, a standard LQR controller for the nominal system is designed ("Nom"). Also, a robust LQR controller will be designed for the system ("Rob"). This is then compared with two controllers designed using the combined controller design technique. One is a controller with one time delay (" 1 TDF") whereas the other is a controller with two time delays ("2 TDF"). Each side of the uncertain hypercube has a length of $2 \sigma=2 \times 0.2$. The parameters of the resulting control profiles have been collected in Table 2. In this table, the feedback controller gains are given as

$$
k^{T}=\left[k_{1}, k_{2}, k_{3}, k_{4}\right]
$$

and the time-delay prefilter's step response is given by

$$
u(t)=\left(1-\sum_{i=1}^{k} A_{i}\right)+\sum_{i=1}^{k} A_{i} \mathcal{H}\left(t-T_{i}\right),
$$

where $\mathcal{H}$ denotes the Heavyside function.

For the time delay filter, the number of switches can be chosen arbitrarely. In general, more delays make the filter more robust, whereas less switches allow for a faster response at the nominal parameter point. For the example presented in this paper and an assumed maximum parameter deviation of $20 \%$ in each direction, a two-delay time delay filter is sufficient to obtain good system performance. The introduction of more delays into the filter decreases the cost only marginally.

Figures 4 and 5 compare the different controllers on the same system. The step responses indicate the superior performance of the hybrid controller since the system comes to rest more quickly and barely displays oscillations. Similar results can be seen for the step responses of the nominal system shown in Figure 4 as well as for a perturbed system. Figure 5 shows the system response for corner number 2 . This corner number is a binary encoded specification of whether the lower bound (bit=0) or upper bound (bit=1) is used. The corner shown in the first diagram is the corner number 2 or $0010_{b i n}$, i. e. the uncertain vector supplied has been $p=\left(p_{L}(1), p_{L}(2), p_{U}(3), p_{L}(4)\right)$.
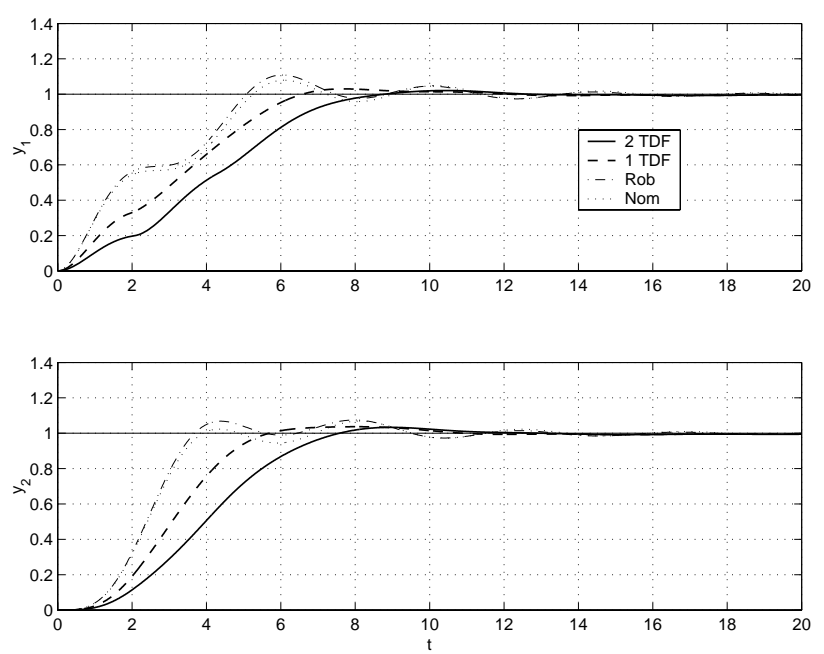

Fig. 4 Step Response (Nominal System), Controller Optimized for $\sigma=20 \%$ and $\alpha=0.9$
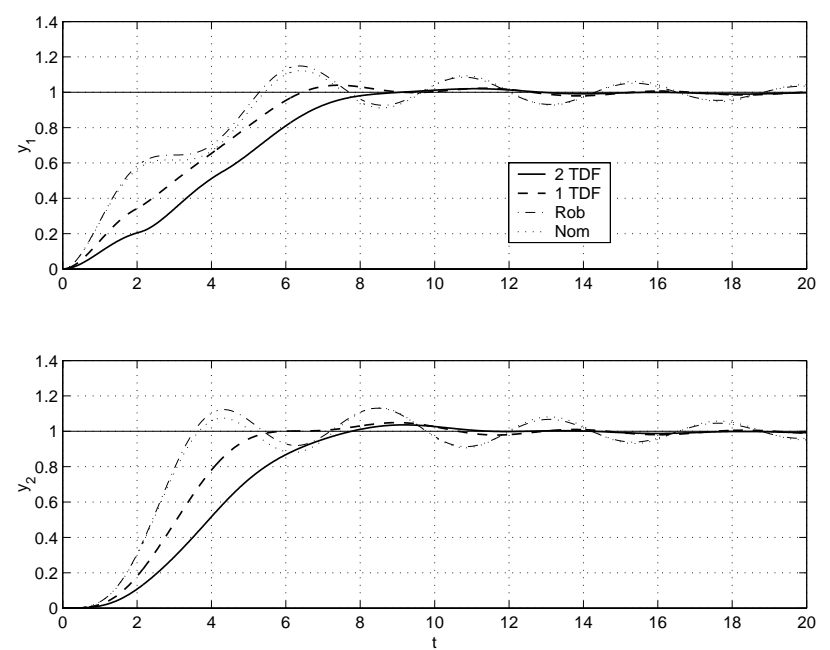

Fig. 5 Step Response (Corner 2), Controller Optimized for $\sigma=20 \%$ and $\alpha=0.9$

Figures 6 and 7 show the control input, which is acting on the system. The diagrams clearly illustrate that 
the control energy expended for the maneuver is also reduced by the use of a time-delay input prefilter. Due to the staircase shape of the prefiltered reference, at $t=0$, the full control is not applied to the system, but only a fraction of it. Later, as the time delayed inputs have propagated, the control acting on the system will be increased. However, since the system is already reacting to the inital control input, the control difference will in general be smaller.

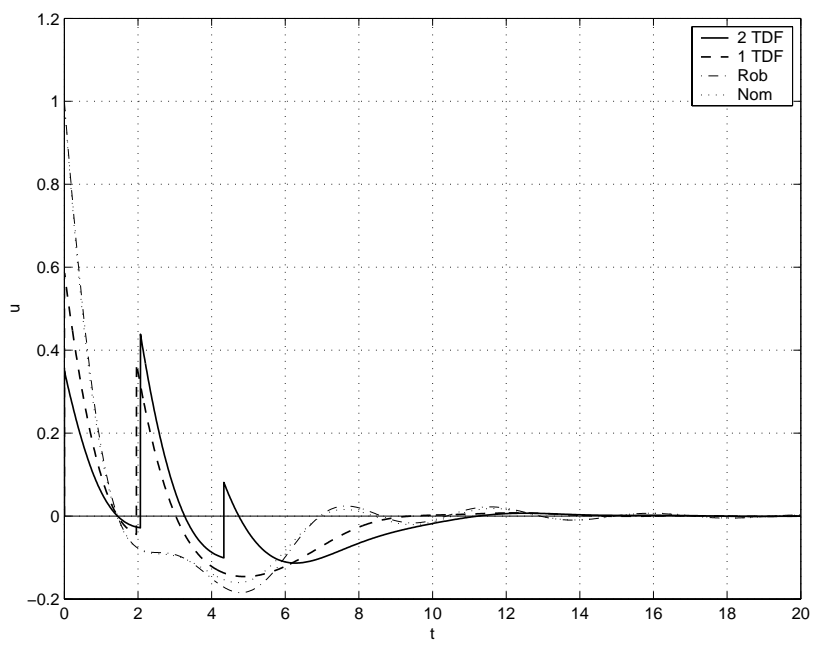

Fig. 6 Control Input (Nominal System), Controller Optimized for $\sigma=20 \%$ and $\alpha=0.9$

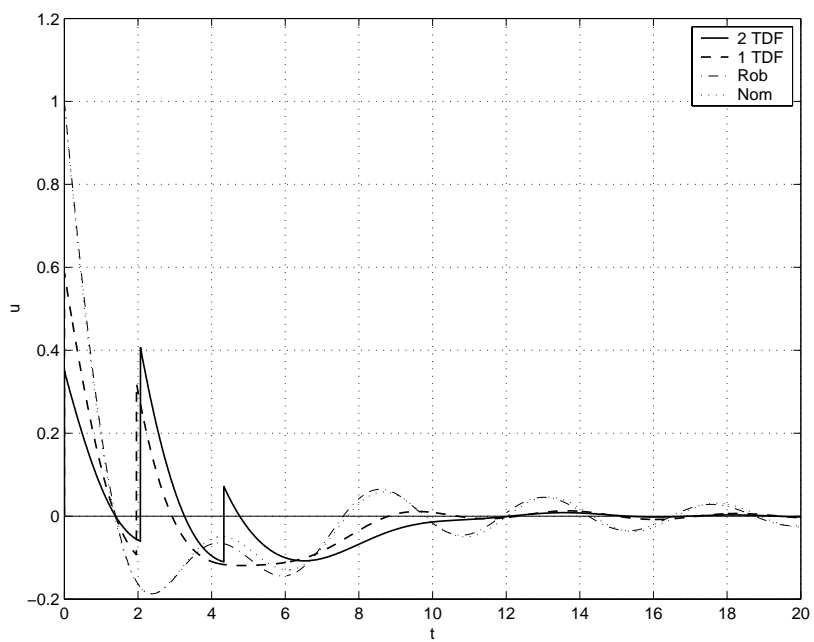

Fig. 7 Control Input (Corner 2), Controller Optimized for $\sigma=20 \%$ and $\alpha=0.9$

As a gauge for the disturbance rejection capabilities, the impulse response has been calculated. This is shown in Fig. 8 and 9. The noise rejection capabilities have hardly been affected. This was expected since the time-delay filter is primarily targeting the step response performance and will by itself not alter the disturbance rejection capabilities. Since the cost function judges both, disturbance rejection and step response characteristics, the gains of the feedback controller will be reasonalby tuned and do not concentrate on the step response only.
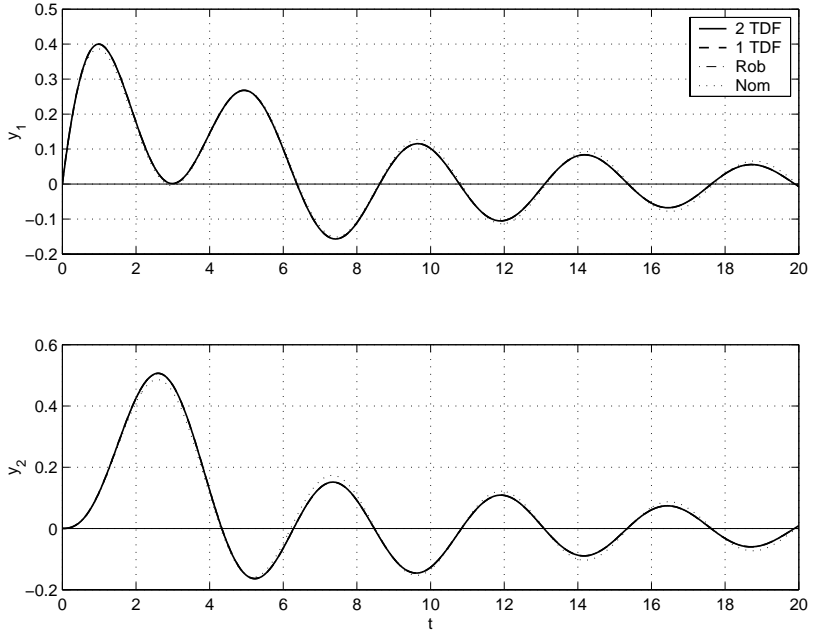

Fig. 8 Impulse Response (Corner 2), Controller Optimized for $\sigma=20 \%$ and $\alpha=0.9$
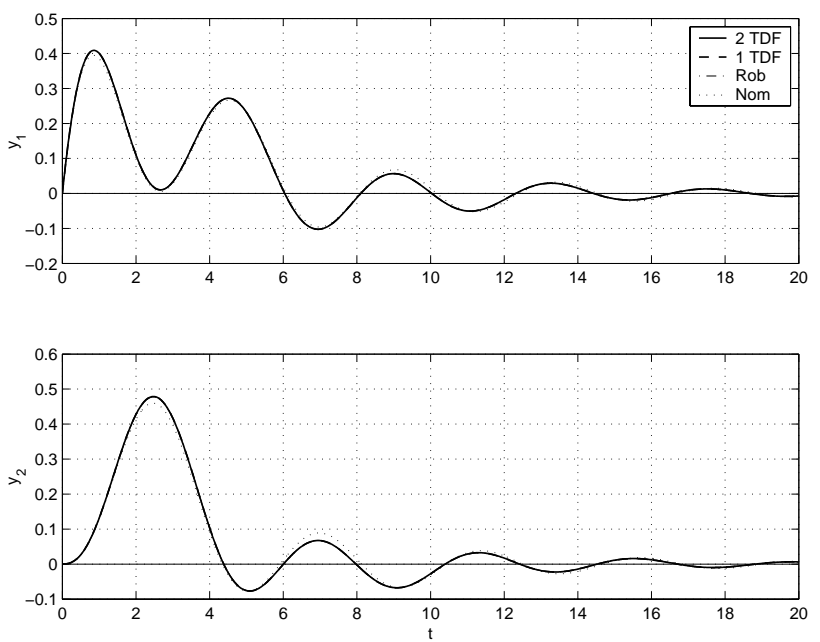

Fig. 9 Impulse Response (Nominal System), Controller Optimized for $\sigma=20 \%$ and $\alpha=0.9$

Figure 10 displays the change in the residual energy. The residual energy is a measure for the input tracking capability. One can clearly see the contribution of the added time-delay filter to the control performance. Figure 11, which graphs the quadratic cost rating the disturbance rejection capabilities, evinces that the performance was not affected by the added time delay filter.

Figure 12 shows the parameter margin for controllers designed with different $\sigma$ bounds. The parameter margin plots the inverse cost of the worst corner over the parameter deviation $\sigma$. Where the curve crosses the $\sigma$ axis, the system becomes unstable. From Fig. 12 it can be seen, that as the variable $\sigma$, which is a metric of uncertainty is increased, the parameter margin increases with a simultaneous decrease in the performance of the nominal system.

The diagrams shown in Fig. 13 and Fig. 14 shows the position of the poles of the closed loop system as the 


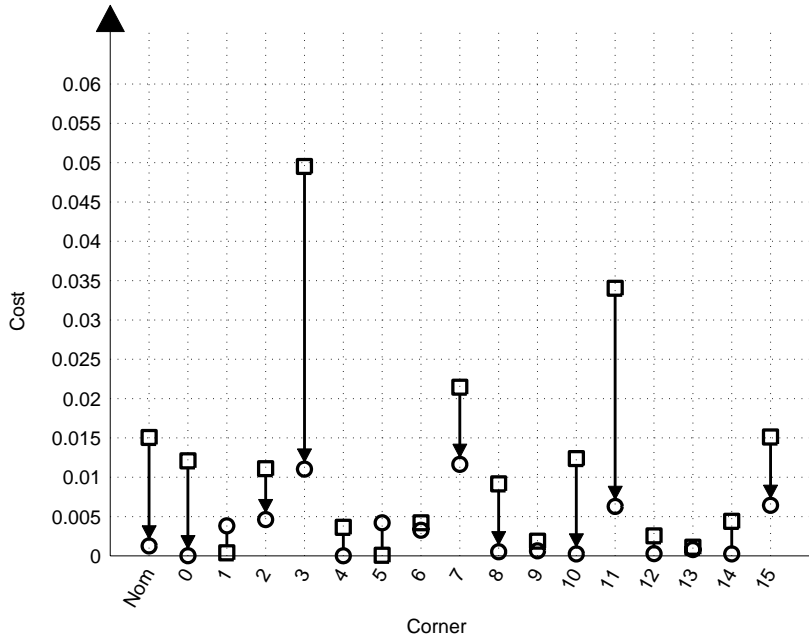

Fig. 10 Change in Residual Energy Cost for $\alpha=0.9$ ( $\square$ with LQR - $\circ$ with LQR and TDF)

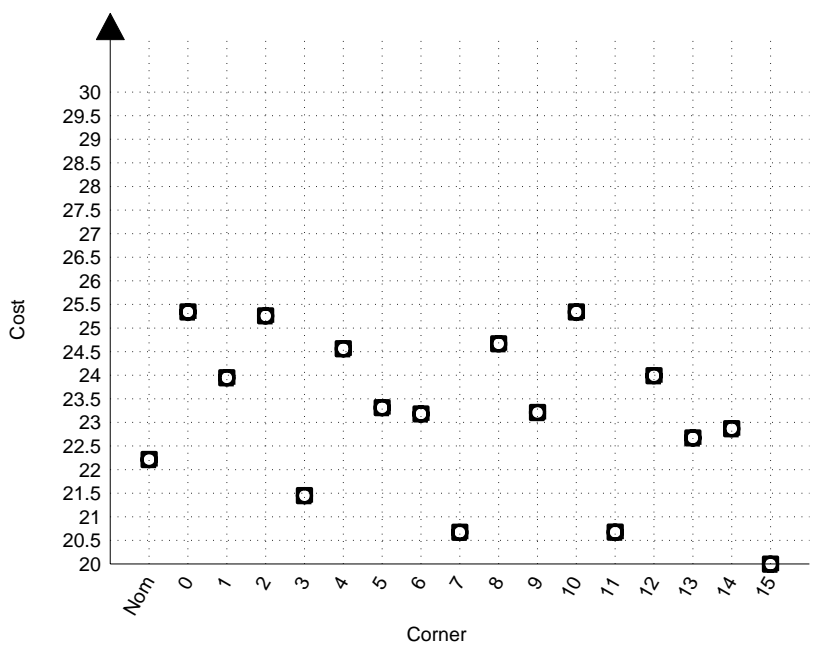

Fig. 11 Change in Quadratic Disturbance Cost for $\alpha=0.9$ ( $\square$ with LQR $-\circ$ with $\mathbf{L Q R}$ and TDF)

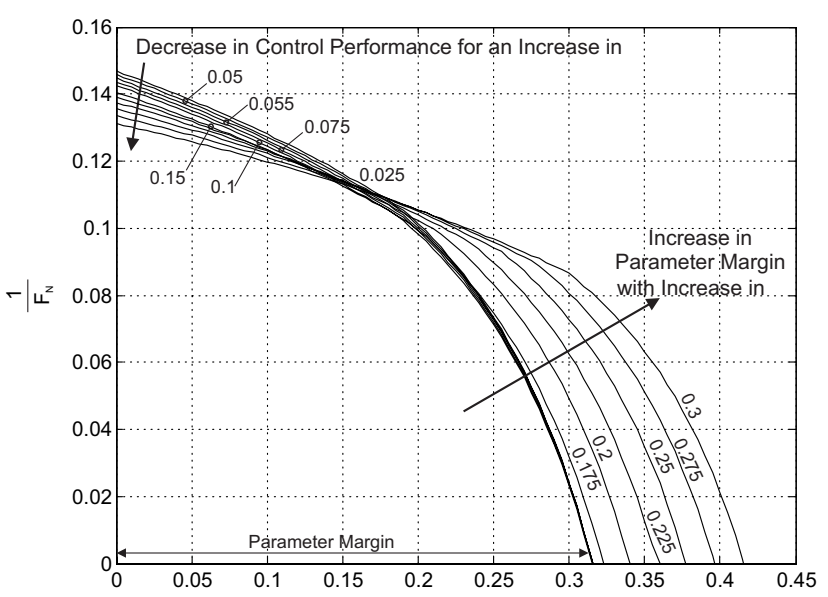

Fig. 12 Parameter Margin for $\alpha=0.9$ as a function of $\sigma$
Table 3 Control Profile Parameters for $\alpha=0.999$ and $\sigma=0.2$

\begin{tabular}{c|c|c|r|r} 
& Nom & Rob & 1 TDF & 2 TDF \\
\hline$k_{1}$ & 0.7669 & 0.8184 & 0.8602 & 0.8429 \\
\hline$k_{2}$ & 0.2331 & 0.1832 & 0.1462 & 0.1642 \\
\hline$k_{3}$ & 1.2298 & 1.1350 & 1.3219 & 1.1609 \\
\hline$k_{4}$ & 1.1226 & 1.0168 & 0.8428 & 0.9659 \\
\hline$T_{1}$ & - & - & 2.1091 & 2.1336 \\
\hline$A_{1}$ & - & - & 0.4511 & 0.4983 \\
\hline$T_{2}$ & - & - & - & 4.2446 \\
\hline$A_{2}$ & - & - & - & 0.1385 \\
\hline
\end{tabular}

parameters are varied. The position of the poles for the nominal system parameters are denoted by "N".

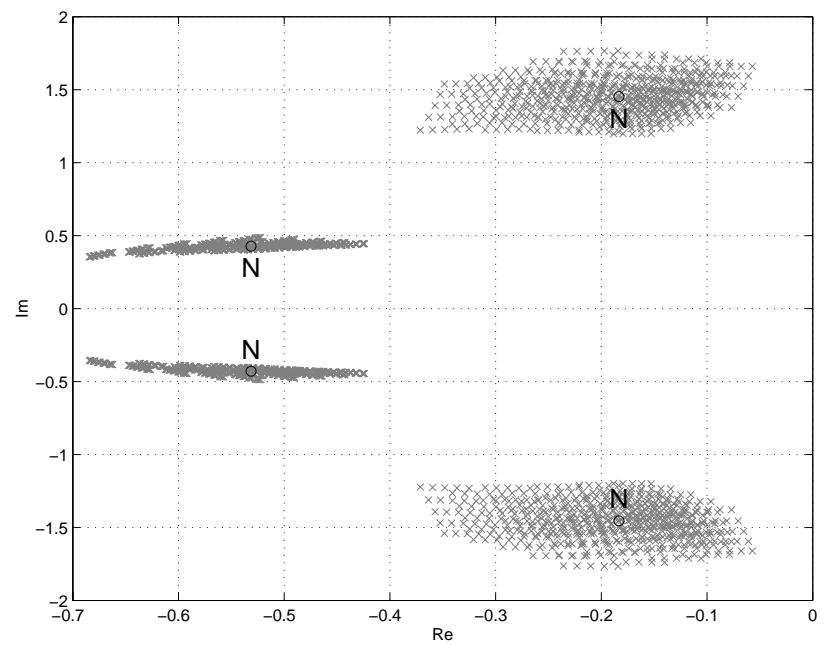

Fig. 13 Position of the Closed-Loop Poles for the Nominal LQR controller

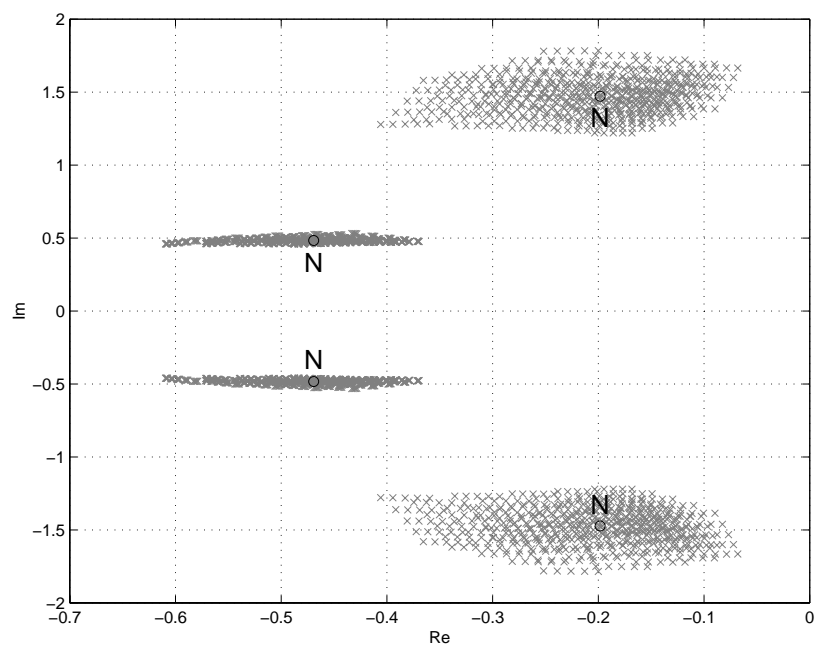

Fig. 14 Position of the Closed-Loop Poles for the Robust LQR controller

To show the effect of the change in $\alpha$ on the controller gains, another controller design has been carried out, this time for a weighting factor of $\alpha=0.999$. The results have been tabulated in Table 3 . 


\section{CONCLUSIONS}

In this paper, a design method for combined feedback/feed-forward controllers was developed. The controller consists of a time delay filter which is augmented by a linear state-feed-back controller. This combination was selected to overcome difficulties typically encountered in the control of flexible structures. The time-delay filter is used to avoid excitation of the oscillatory modes of the flexible structure. The feedback controller, which is a linear state feed-back controller is used to damp out any remaining vibrations which could originate from disturbances or parametric uncertainties.

The controller is designed using a minimax optimization scheme. This permits accounting for parametric uncertainties and including statistical information such as mean values, lower and upper bounds. The algorithm first designs both controller for the nominal system using well understood methods. Then, the optimization program will look at a hypercube in the uncertain space which is repeatedly increased until the entire uncertain space is spanned. The cost function is a weighted sum rating both control tracking and disturbance rejection. For all cost functions, analytical gradients are supplied.

Numerical examples show the feasibility of the proposed design approach and also illustrate the increase in control performance which could be gained. The system under consideration for all examples is the Floating Oscillator, a standard benchmark problem.

\section{ACKNOWLEDGEMENTS}

This work was completed during the second author's sabbatical stay at the Technical University of Darmstadt under the sponsorship of the von Humboldt Stiftung. The second author gratefully acknowledges their support.

\section{References}

${ }^{1}$ Junkins, J. L., Rahman, Z., and Bang, H., NearMinimum Time Maneuvers of Flexible Vehicles: A Liapunov Control Law Design Method, Mechanics and Control of Large Flexible Structures, AIAA Publication, Washington, DC., 1990.

${ }^{2}$ Ballhaus, W. L., Rock, S. M., and Bryson, A. E., "Optimal Control of a Two-Link Flexible Robotic Manipulator Using Time-Varying Controller Gains," Amer. Astronautics Soc. paper 92-055, 1992.

${ }^{3}$ Bhat, S. P. and Miu, D. K., "Minimum Power and Minimum Jerk Control and its Application in Computer Disk Drives," IEEE Transactions on Magnetics, Vol. 27, No. 6, 1991, pp. 4471-4475.

${ }^{4}$ Singhose, W. E., Porter, L. J., and Seering, W. P., "Input Shaped Control of a Planar Gantry Crane with Hoisting," 1997 American Control Conference, 1997.
${ }^{5}$ Singhose, W., Singh, T., and Seering, W., "OnOff Control with Specified Fuel Usage," ASME Journal of Dynamic Systems, Measurement and Control, Vol. 121, No. 2, 1999, pp. 206-212.

${ }^{6}$ Singer, N. C. and Seering, W. P., "Preshaping Command Inputs to Reduce System Vibrations," ASME Journal of Dynamic Systems, Measurement and Control, Vol. 115, 1990, pp. 76-82.

${ }^{7}$ Singh, T. and Vadali, S. R., "Robust Time-Delay Control of Multimode Systems," International Journal of Control, Vol. 62, No. 6, 1993, pp. 1319-1339.

${ }^{8}$ Banerjee, A. K. and Singhose, W. E., "Minimum Time Fuel Efficient Maneuver of Flexible Spacecraft with Vibration Amplitude Constraint," AAS Astrodynamics Specialist Conference, Vol. AAS 95-318, Halifax, Nova Scotia, 1995.

${ }^{9}$ Smith, O. J. M., "Posicast Control of Damped Oscillatory Systems," Proceedings of the IRE, 1957, pp. 1249-1255.

${ }^{10}$ Singh, T., "Minimax Design of Robust Controllers for Flexible Structures," To appear in the AIAA Journal of Guidance, Control and Dynamics, 2002.

${ }^{11}$ Muenchhof, M. and Singh, T., "Desensitized Jerk Limited Time Optimal Control of Multi-Input Systems," Journal of Guidance, Control and Dynamics, Vol. 25, No. 3, 2002, pp. 474-481.

${ }^{12}$ Singh, T. and Singhose, W., "Tutorial on Input Shaping/Time Delay Control of Maneuvering Flexible Structures," American Control Conference 2002, Anchorage, Alaska, 2002.

${ }^{13}$ Dorato, P., Abdallah, C., and Cerone, V., Linear Quadratic Control: An Introduction, Prentice-Hall, Englewood Cliffs, NJ, 1995.

${ }^{14}$ Mills, R. and Bryson, A., "Parameter-Robust Control Design Using a Minimax Method," Journal of Guidance, Control, and Dynamics, Vol. 15, No. 5, 1992, pp. 1068-1075.

${ }^{15}$ Mills, R. and Bryson, A., "Linear-QuadraticGaussian Controllers with Specified Parameter Robusteness," Journal of Guidance, Control, and Dynamics, Vol. 21, No. 1, 1998, pp. 1068-1075.

${ }^{16}$ Kapila, V., Tzes, A., and Qiguo, Y., "Closed-Loop Input Shaping for Flexible Structures Using TimeDelay Control," ASME Journal of Dynamic Systems, Measurement and Control, Vol. 122, 2000, pp. 454460.

${ }^{17} \mathrm{Wie}$, B. and Bernstein, D., "Benchmark Problems for Robust Control Design," Journal of Guidance, Control, and Dynamics, Vol. 15, No. 5, 1992, pp. 10571058. 